\title{
Considerações sobre o âng'ulo de administração de colírios antiglaucomatosos análogos das prostaglandinas
}

\author{
Considerations about administration angle of prostaglandin analogs
}

\author{
Paulo Estacia ${ }^{1}$ \\ Taíse Tognon ${ }^{2}$
}

Trabalho realizado na Universidade de Passo Fundo Passo Fundo (RS) - Brasil.

${ }^{1}$ Médico oftalmologista, Mestre e Doutor, Professor da Disciplina de Oftalmologia da Faculdade de Medicina da Universidade de Passo Fundo - UPF - Passo Fundo (RS) - Brasil.

${ }^{2}$ Acadêmica de Graduação em Medicina da Universidade de Passo Fundo - UPF - Passo Fundo (RS) - Brasil. Endereço para correspondência: Paulo Estacia. Rua Teixeira Soares, 885/502 - Passo Fundo (RS) CEP 99010-081

E-mail: paulo@estacia.trix.net

Recebido para publicação em 07.11.2007

Última versão recebida em 31.05.2008

Aprovação em 08.06.2008

Nota Editorial: Depois de concluída a análise do artigo sob sigilo editorial e com a anuência da Dra. Christiane Rolim de Moura sobre a divulgação de seu nome como revisora, agradecemos sua participação neste processo. Conflito de Interesse: Os autores não possuem interesses comerciais ou de propriedade nos produtos utilizados na elaboração deste trabalho.

\section{RESUMO}

Objetivo: Investigar se há diferença no peso e volume das gotas de colírios análogos das prostaglandinas em ângulos de gotejamento de $45^{\circ}$ e $90^{\circ}$ com relação ao plano horizontal. Métodos: Foi realizado estudo experimental utilizando os colírios latanoprosta, travoprosta e bimatoprosta, pelo qual se gotejava as soluções com angulação de $45^{\circ}$ e $90^{\circ}$. Estes colírios foram escolhidos em virtude do seu uso rotineiro em oftalmologia e do seu custo. A primeira gota e dez gotas seguintes foram pesadas em ângulo de $45^{\circ}$ e $90^{\circ}$. A análise estatística foi realizada por meio do programa SPSS ${ }^{\circledR} 12.0$ (Microsoft), utilizando o teste de variância ANOVA, sendo considerada diferença estatisticamente significante um valor $P<0,001$. Resultados: Verificou-se que há diferença no peso, e conseqüentemente no volume, das gotas instiladas a $45^{\circ}$ e a $90^{\circ}$ dos colírios de travoprosta e bimatoprosta. Para o colírio travoprosta o gotejo a $45^{\circ}$ produz uma gota menor que em $90^{\circ}$. O inverso ocorre para o colírio de bimatoprosta. Já para o colírio de latanoprosta, não houve diferença estatisticamente significante. Conclusões: Como houve diferença estatística no peso das gotas de dois colírios análogos das prostaglandinas e em outro não se verificou esta variação, - e como este possui relação direta com o volume - infere-se que devemos ser críticos diante de estimativas de custo ou duração da terapia com base no gotejamento dos colírios considerando número de gotas por frasco e volume.

Descritores: Glaucoma/quimioterapia; Preço de medicamento; Soluções oftálmicas/economia; Soluções oftálmicas/administração \& dosagem; Instilação de medicamentos; Prostanglandinas F sintéticas/administração \& dosagem; Custos de cuidados de saúde; Gastos em saúde; Estudo comparativo

\section{INTRODUÇÃO}

Glaucoma é uma designação genérica para um grupo de diversas condições oculares que têm em comum a neuropatia óptica progressiva ou anormalidades na drenagem do humor aquoso na câmara anterior ${ }^{(1)}$.

Embora algumas formas clínicas de glaucoma possuam manifestações agudas e proeminentes, a maioria dos pacientes tem uma doença crônica que se desenvolve de modo lento, apresenta maior incidência com o aumento da idade e não produz sintomas até que o dano no nervo óptico se instale e a perda de campo visual seja $\operatorname{avançada~}^{(1)}$.

A decisão de iniciar o tratamento do glaucoma deve ser individualizada para cada paciente, e uma vez iniciada a terapêutica, o paciente geralmente terá que continuá-la durante toda a vida. Neste sentido, os pacientes 
devem ser alertados sobre efeitos colaterais adversos, custos significativos, mudanças na rotina diária e na qualidade de vida devido ao uso de medicações antiglaucomatosas ${ }^{(2-3)}$.

Uma vez que o aumento da pressão intra-ocular parece ser o fator de risco principal para o desenvolvimento de lesões no glaucoma, na maioria dos casos o tratamento com colírios hipotensores oculares é a primeira terapia instituída ${ }^{(4)}$.

Os análogos das prostaglandinas são uma classe de medicamentos relativamente recente. Eles derivam do ácido araquidônico e desempenham uma série de funções biológicas, entre as quais o aumento do fluxo úveo-escleral e são muito prescritos para tratamento desta condição em virtude de sua eficácia e do conforto de sua posologia ${ }^{(2-3)}$.

Como em toda doença crônica, o paciente com glaucoma apresenta problemas de fidelidade ao tratamento, que é influenciada por vários fatores. O custo, em especial dos análogos das prostaglandinas, é um dos fatores relevantes na escolha de uma medicação. No cálculo das despesas destas novas medicações antiglaucomatosas deve-se considerar não apenas o preço real do colírio na farmácia, mas o valor diário da terapia baseado no volume da gota, volume do frasco, dosagem da administração, eficácia do uso e o gasto relacionado ao tratamento ${ }^{(5-6)}$.

$\mathrm{O}$ volume da gota instilada está diretamente relacionado à biodisponibilidade da droga, a fim de proporcionar uma quantidade padrão e adequada para ação do fármaco. As apresentações comerciais mais antigas de colírios apresentavam volumes que variam entre 50 e $75 \mathrm{ml}$ e atualmente os frascos fornecem gotas variando entre 25 e $56 \mathrm{ml}^{(7-8)}$. Também o ângulo no qual o colírio é administrado constitui uma das variáveis que atua modificando o volume da $\operatorname{gota}^{(9)}$.

É importante produzir uma gota menor com maior concentração da droga, pois a ação farmacológica depende da quantidade residual em contato com a superfície ocular após perdas por diluição, ligação e drenagem ${ }^{(7)}$. A melhor concentração em filme lacrimal é atingida com uma gota de $20 \mathrm{ml}$, sendo que o aumento do volume da gota aumenta a absorção sistêmica, o desperdício e pode piorar a tolerabilidade ${ }^{(10-11)}$.

Variações amplas no número de gotas por frasco com o mesmo volume, mas de drogas diferentes, têm sido reportadas em vários estudos, assim como diferença na contagem do número de gotas entre vários frascos de um mesmo produto ${ }^{(12)}$.

O número de gotas por mililitro, o volume verdadeiro, e o custo por frasco são usuais para determinar o custo diário com o tratamento em pacientes com glaucoma.

Vários trabalhos têm sido publicados sobre o custo do tratamento antiglaucomatoso com colírios, com base no número de gotas obtido de cada frasco ${ }^{(5,8,11-16)}$.

É importante observar que a densidade das soluções oftalmológicas não tem papel significante na determinação do volume da gota ${ }^{(17)}$. Daí o fato de que o peso da gota possui relação direta com seu volume, e conseqüentemente com seu tamanho. Isto parece ser devido ao fato de que a densidade dos líquidos acondicionados nos colírios é aproximadamente uniforme ${ }^{(17)}$.
Frente ao exposto, o objetivo desta pesquisa consiste em investigar se há diferença no peso e no volume das gotas de colírios análogos das prostaglandinas instiladas quando se adota ângulos de gotejamento de $45^{\circ}$ e $90^{\circ}$ com relação ao plano horizontal.

\section{MÉTODOS}

A pesquisa consiste em um estudo experimental, realizado nos laboratórios do Instituto de Ciências Biológicas da Universidade de Passo Fundo. Foram utilizados 3 frascos originais de cada um dos colírios antiglaucomatosos do tipo análogo das prostaglandinas, a saber: latanoprosta $0,005 \%$ (Xalatan ${ }^{\circledR}$, Pfizer), travoprosta $0,004 \%$ (Travatan $^{\circledR}$, Alcon) e bimatoprosta $0,03 \%$ (Lumigan ${ }^{\circledR}$, Allergan), de 2,5 ml (latanoprosta e travoprosta) e $1,5 \mathrm{ml}$ (bimatoprosta). Os colírios representantes destas marcas possuíam diferentes frascos e gotejadores. Os colírios análogos das prostaglandinas foram os escolhidos em virtude do seu uso cada vez mais freqüente e pelo seu custo elevado.

Os frascos foram adquiridos em farmácias aleatoriamente escolhidas. O número do lote de cada colírio foi arquivado. Consideramos que o volume de cada frasco era o mesmo informado na embalagem e que o peso das gotas correlacionava-se diretamente com seu volume.

Todo o experimento foi realizado em temperatura ambiente de $22^{\circ} \mathrm{C}$ e para sua realização utilizou-se uma balança de precisão - Ohaus Explorer ${ }^{\circledR}$ Balance E02140 -, com capacidade métrica de $210 \mathrm{~g}$, sensibilidade e reprodutibilidade de até $0,1 \mathrm{mg}$, em temperatura ambiente $\left(10^{\circ} \mathrm{C}\right.$ a $\left.30^{\circ} \mathrm{C}\right)$ e calibrada a cada medida, para cada frasco.

A contagem de gotas de todos os frascos foi realizada por um único investigador. Todos os colírios tiveram gotas instiladas com o ângulo de $90^{\circ}$ e de $45^{\circ}$, sendo este ângulo de administração determinado pela relação entre a base do frasco e um plano de referência.

Cada colírio era aberto, seu lote era anotado e seguia-se então para o processo de pesagem. A primeira gota e dez gotas subseqüentes eram pesadas em ângulo de $45^{\circ}$ e após realizava-se o mesmo processo com o ângulo em $90^{\circ}$. Cada frasco foi delicadamente pressionado para instilação de somente uma gota. Um intervalo de cerca de 10 segundos era observado entre cada gota e o frasco era colocado na vertical entre cada gotejo, a fim de simular o processo realizado pelo paciente durante a instilação do colírio. A escolha do colírio era aleatória. A cada dez gotas o recipiente de pesagem era trocado e a balança era calibrada com graduação de $0,1 \mathrm{mg}$. Todos os recipientes para a pesagem eram iguais. Esse procedimento foi repetido até o término do conteúdo dos colírios.

Análise estatística foi efetuada em SPSS ${ }^{\circledR} 12.0$ (Statistical Package for Social Sciences, Microsoft). A comparação das variáveis quantitativas analisadas foi procedida através do teste de variância ANOVA, sendo considerada uma diferença estatisticamente significante um valor de probabilidade $(P)$ inferior a 0,001 . 


\section{RESULTADOS}

Após a realização do gotejamento tanto em $90^{\circ}$ como em $45^{\circ}$ com relação ao plano horizontal, calculou-se o peso médio de cada gota (com base em grupos de 10 gotas) nas duas angulações, bem como o desvio padrão, que segue na tabela 1 .

Para fins de comparação, utilizou-se teste de comparações múltiplas (Tukey-Kramer), adotando-se intervalo de confiança $>95 \%$ e observou-se que as três marcas possuíam peso, e conseqüentemente volume, de gotas instiladas diferentes entre si.

Após análise estatística de variância utilizando teste ANOVA, e adotando-se $P<0,001$, verificou-se que há diferença estatisticamente significativa no peso das gotas instiladas a $45^{\circ}$ e a $90^{\circ}$ dos colírios travoprosta e bimatoprosta. Tem-se que para o colírio de travoprosta o gotejo a $45^{\circ}$ produz uma gota menor do que a $90^{\circ}$, como pode ser verificado pela análise do gráfico 1.

Para o colírio de bimatoprosta ocorre o fenômeno inverso, a menor gota ocorre com instilação a $90^{\circ} \mathrm{em}$ relação ao plano horizontal $(P<0,001)$, gráfico 2 .

No gráfico 3 é possível se visualizar, por outro lado, que para o colírio análogo da prostaglandina latanoprosta, não houve diferença estatisticamente significante no tamanho da gota, independentemente da angulação utilizada para a instilação $(P=0,212)$.

\begin{tabular}{|lcc|}
\hline \multicolumn{3}{|l|}{ Tabela 1. Colírios antiglaucomatosos e peso médio para $\mathbf{1 0}$ gotas } \\
Produto & $\begin{array}{c}\text { Média peso } \mathbf{( m g )} \\
\mathbf{1 0} \text { gotas a } \mathbf{4 5}^{\circ}\end{array}$ & $\begin{array}{c}\text { Média peso } \mathbf{( m g )} \\
\mathbf{1 0} \text { gotas a } \mathbf{9 0}^{\circ}\end{array}$ \\
Travoprosta & $0,259917 \pm 0,010715$ & $0,299117 \pm 0,005241$ \\
Latanoprosta & $0,326167 \pm 0,005422$ & $0,320050 \pm 0,009828$ \\
Bimatoprosta & $0,292350 \pm 0,007085$ & $0,269333 \pm 0,008221$ \\
\hline
\end{tabular}

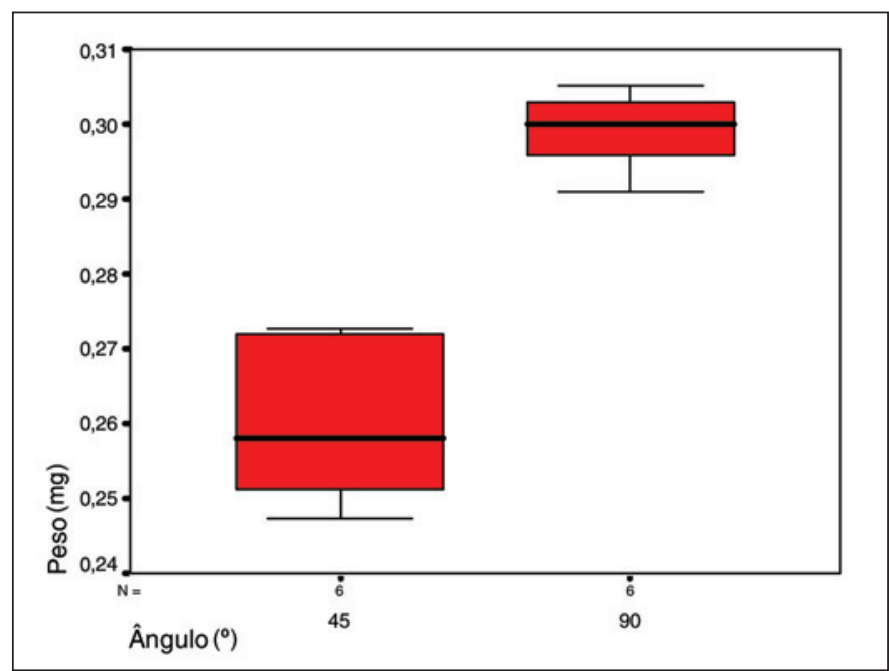

Gráfico 1 - Peso (10 gotas em $\mathrm{mg}$ ) de acordo com gotejo em $45^{\circ}$ e $90^{\circ}$ para travoprosta

\section{DISCUSSÃO}

É sabido que o glaucoma constitui uma patologia importante do ponto de vista social e populacional, em virtude de sua morbidade e custos ao sistema de saúde e ao paciente. Assim, é indiscutível que a terapêutica uma vez instituída deve ser efetiva e, a mais empregada, como descrito anteriormente, é o uso de colírios antiglaucomatosos. Para tanto, a gota instilada deve possuir um volume adequado para o que se deseja. O olho humano pode acomodar uma gota de colírio com volume não superior a $23 \mathrm{ml}$ e quantidades superiores a esta são desperdiçadas, ou são drenadas pela via lacrimal com possibilidade de maior absorção sistêmica ${ }^{(18)}$.

Estudos anteriores mostraram que a média de volume das gotas de soluções oftálmicas era de $39 \mathrm{ml}$ com uma variação de

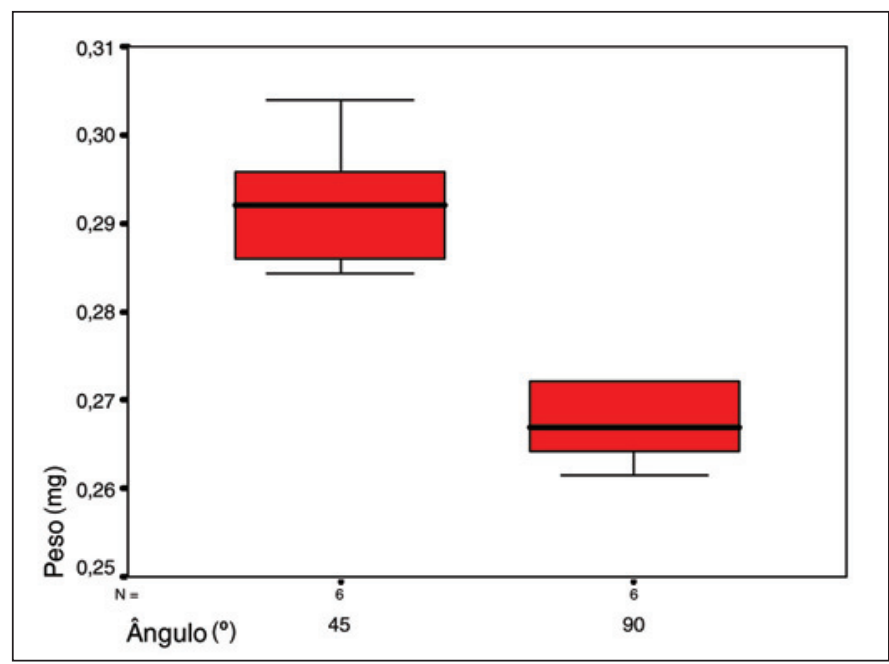

Gráfico 2 - Peso (10 gotas em $\mathrm{mg}$ ) de acordo com gotejo em $45^{\circ}$ e $90^{\circ}$ para bimatoprosta

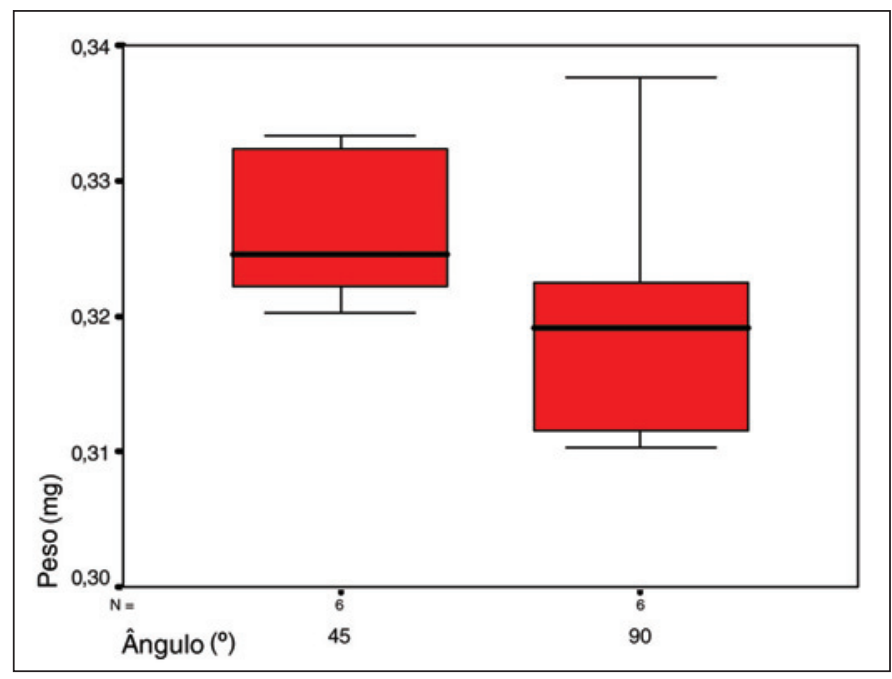

Gráfico 3 - Peso (10 gotas em $\mathrm{mg}$ ) de acordo com gotejo em $45^{\circ}$ e $90^{\circ}$ para latanoprosta 
25,1 a $56,4 \mathrm{ml}^{(19)}$. Em nosso país o Ministério da Saúde preconiza que as gotas de colírios devem apresentar volumes inferiores a $50 \mathrm{ml}$. Assim, observa-se que todos os diferentes produtos testados possuíam correlação com o estudo citado.

Como a densidade das soluções usadas nos colírios é aproximadamente constante, deve-se ressaltar o aspecto de que o peso da gota possui relação direta com seu volume e seu tamanho, sendo então proposto que a densidade da gota ocular tem papel ínfimo na determinação do seu volume ${ }^{(17)}$.

Entre os fatores a serem considerados para explicar a diferença de tamanho nas gotas dos frascos das três marcas de colírios em angulações diferentes estão a tensão de superfície e o diâmetro externo do orifício do gotejador ${ }^{(17)}$. Esta relação é dada pela lei de Tate, que descreve que o peso da gota é diretamente proporcional à tensão superficial do líquido e o diâmetro da ponta. Líquidos com maior tensão superficial em gotejadores com orifícios de maior diâmetro externo, não arredondados, formariam uma gota de tamanho maior quando o frasco estivesse a $90^{\circ}$. Por outro lado, inclinação do frasco para $45^{\circ}$ diminuiria a área de contato do líquido com a superfície externa do orifício do gotejador e, com auxílio da força da gravidade, formaria uma gota menor. Ao contrário, um líquido com tensão superficial menor, em um frasco com gotejador arredondado, de orifício externo pequeno, formaria uma gota maior a $45^{\circ}$ porque este líquido não mantém coesão e se espalharia pela área arredondada do gotejador, ampliando a área de superfície da ponta.

Os gotejadores dos frascos de travoprosta e bimatoprosta apresentam características construtivas que se correlacionam, respectivamente, com a descrição acima, e os resultados obtidos são compatíveis com o design dos colírios.

Ainda, outros autores também descreveram que as diferenças nos bicos gotejadores têm uma influência decisiva no tamanho da gota administrada ${ }^{(9)}$.

As embalagens das medicações analisadas não variavam somente quanto ao seu bico gotejador, mas também no formato e no material de fabricação dos frascos.

Também deve ser considerado que o volume das gotas pode variar nos diferentes lotes da medicação analisada ${ }^{(6)}$.

Toda a discussão acerca dos tamanhos de gotas tem a finalidade de proporcionar diminuição dos custos para o paciente, uma vez que todas as medicações antiglaucomatosas investigadas nesta pesquisa possuem a mesma posologia.

Os oftalmologistas são apresentados freqüentemente a gráficos e tabelas das diferentes indústrias de colírios antiglaucomatosos, mostrando que seu produto possui menor custo ao consumidor. Como apurado em nosso estudo, essa afirmativa somente é válida quando se especifica o procedimento de gotejamento adotado para a administração deste, sendo que diferentes metodologias produzem resultados que beneficiam determinados colírios. Ainda, existem trabalhos que analisaram custos de medicação antiglaucomatosa, porém adotaram diferentes métodos ou não descreveram em seus estudos a angulação em que o gotejo foi realizado, o que pode confundir a interpretação dos resultados ${ }^{(5,8,11-16)}$.

\section{CONCLUSÃO}

Verificamos que o peso, e conseqüentemente o tamanho, das gotas de colírios antiglaucomatosos análogos das prostaglandinas instiladas varia conforme a metodologia empregada para os frascos de dois fármacos: travoprosta e bimatoprosta. As gotas de travoprosta são menores quando administradas a $45^{\circ}$ e as gotas de bimatoprosta são menores quando administradas a $90^{\circ}$ com relação ao plano horizontal. Não houve diferença estatisticamente significativa no volume das gotas instiladas tanto a $45^{\circ}$ quanto a $90^{\circ}$ do colírio latanoprosta.

Assim, infere-se que para a análise de custos com base no número de gotas e no rendimento de cada frasco a metodologia empregada deve ser relatada, uma vez que o resultado final pode favorecer determinado produto.

Ainda, os oftalmologistas devem ser críticos quando apresentados a planilhas ou estudos sobre a variação de custos de colírios para o consumidor, pois pode haver variação no volume das gotas conforme o método de gotejamento.

\section{AGRADECIMENTOS}

Os autores agradecem os professores Adil Pacheco e Adriano Pasqualotti, da Universidade de Passo Fundo/RS, por colaborarem na realização desta pesquisa.

\section{ABSTRACT}

Purpose: To investigate if there is any difference in volume and drop weight of prostaglandin analogs when adopting drip angles of $45^{\circ}$ and $90^{\circ}$, regarding a horizontal line. Methods: An experimental study was conducted using the follow ophthalmic solutions: latanoprost, travoprost and bimatoprost. In this study the ophthalmic solutions were dripped according to an angle of $45^{\circ}$ or $90^{\circ}$. Prostaglandin analogs were chosen due to their common use in ophthalmology and their cost. The first drop and other ten drops were weighed, alternating the drip angle $\left(45^{\circ}\right.$ or $\left.90^{\circ}\right)$. Statistical analysis was done with $\operatorname{SPSS}^{\circledR} 12.0$ (Microsoft), using quantitative comparisons with the ANOVA test. An odds value $(P)$ below 0.001 was considered a statistical significant difference. Results: We verified differences in weight and size of the travoprost and bimatoprost drops instilled at $45^{\circ}$ and $90^{\circ}$. The drip at $45^{\circ}$ produces a smaller drop of travoprost $(P<0.001)$, and the inverse occurs for bimatoprost. There were no statistical significant differences in weight of latanoprost drops according to the instillation angle. Conclusions: Once there was statistical difference in weight - which has direct relationship with size - of the drops of two kinds of prostaglandin analogs and in another one we did not observe this variation, we infer that cost and therapy duration estimates should be analyzed carefully, especially if this kind of drip measure is used. 
Keywords: Glaucoma/drug therapy; Drug price; Ophthalmic Solutions/economic; Ophthalmic solutions/administration \& dosage; Instillationm, drug; Prostaglandins F, synthetic/administration \& dosage; Health care costs; Health expenditures; Comparative study

\section{REFERÊNCIAS}

1. Fraser S, Wormald R. Epidemiology of glaucoma. In: Yanoff D, Duker JS, editors. Ophthalmology. $2^{\text {nd }}$ ed. St. Louis: Mosby; 2004. p.1413-7.

2. Migdal C. Which therapy to use in glaucoma. In: Yanoff D, Duker JS, editors. Ophthalmology. $2^{\text {nd }}$ ed. St. Louis: Mosby; 2004. p.1539-42.

3. Gross RL. Current medical management of glaucoma. In: Yanoff D, Duker JS, editors. Ophthalmology. $2^{\text {nd }}$ ed. St. Louis: Mosby; 2004. p.1543-52.

4. Zimmerman R, Sakiyalak O, Krupin T, Rosemberg L. Primary open-angle glaucoma. In: Yanoff D, Duker JS, editors. Ophthalmology. 2nd ed. St. Louis: Mosby; 2004. p.1482-7.

5. Pedroso L, Carvalho Jr ES, Paranhos Jr A, Prata Jr JÁ, Mello PA. Custo real do tratamento do glaucoma para o paciente. Arq Bras Oftalmol. 1999;62(6): 677-82.

6. Fiscella RG, Geller JL, Gryz LL, Wilensky J, Viana M. Cost considerations of medical therapy for glaucoma. Am J Ophthamol. 1999;128(4):426-33.

7. Roizenblatt R, Freitas D, Belfort Junior R, Hofling-Lima AL, Prata Jr JA. Impacto econômico no tratamento do glaucoma: volume de gotas de colírios antiglaucomatosos brasileiros e norte-americanos. Arq Bras Oftalmol. 2001; 64(2):143-6
8. Prata Jr JA, Prata JA. Comparação do volume da gota e custo do tratamento dos genéricos de maleato de timolol 0,5\%. Arq Bras Oftalmol. 2004;67(3):419-22.

9. Van Santvliet L, Ludwig A. Determinants of eye drop size. Surv Ophthamol. 2004;49(2):197-213.

10. Nagataki S, Mishima S. Pharmacokinetics of instilled drugs in the human eye. Int Ophthamol Clin. 1980;20(3):33-49.

11. Ventura MP, Saheb NE, Solari HP, Saraiva VS, Vianna RN, Burnier Jr MN. Cost considerations of the new fixed combinations for glaucoma medical therapy. J Clin Pharm Ther. 2005;30(3):251-4.

12. Stillitano IG, Tenório A, Cardoso G, Ribeiro MP, Figueiroa JN. Custo do tratamento de drogas antiglaucomatosas: latanoprost, travoprost, bimatoprost e unoprostona isopropílica. Arq Bras Oftalmol. 2003;66(6):859-64.

13. Stillitano IG, Lima MG, Ribeiro MP, Cabral J, Brandt CT. Impacto econômico do custo de colírios no tratamento do glaucoma. Arq Bras Oftalmol. 2005;68(1):79-84

14. Fiscella RG, Green A, Patuszynski DH, Wilensky J. Medical therapy cost considerations for glaucoma. Am J Ophthamol. 2003;136(1):18-25.

15. Ikeda H, Sato E, Kitaura T, Fukuchi H, Kimura Y, Kihira K. Daily cost of ophthalmic solutions for treating glaucoma in Japan. Jpn J Ophthamol. 2001; 45(1):99-102.

16. Amaral Filho JM, Moreira RR, Silva LM, Vasconcelos JP, Rocha EM, Costa VP, et al. Custo mensal de medicações anti-glaucomatosas no Brasil. Arq Bras Oftalmol. 1999;62(2):123-6.

17. Gaynes BI, Singa RM, Schaab G, Sorokin Y. Impact of administration angle on the cost of artificial tear solutions: does bottle positioning minimize wastage? J Ocul Pharmacol Ther. 2007;23(2):196-201.

18. Vaidergorn PG, Malta RF, Borges AS, Menezes MD, Trindade ES, Malta JB Técnica da instilação de colírios em pacientes portadores de glaucoma crônico. Arq Bras Oftalmol. 2003;66(6):865-9.

19. Lederer C Jr, Harold RE. Drop size of commercial glaucoma medications. Am J Ophthalmol. 1986;101(6):691-4

\title{
ABO Eletrônico \\ A versão eletrônica dos Arquivos Brasileiros de Oftalmologia com textos completos está disponível em
}

\author{
ABO - Arquivos Brasileiros de Oftalmologia \\ http://www.abonet.com.br
}

SCIELO - Scientific Electronic Library Online

http://www.scielo.org

Free Medical Journals

http://www.freemedicaljournals.com

PubMed

http://www.pubmed.com 